\section{SECTION 8}

Gene structure and regulation

\subsection{1}

The structures of members of the glycerol kinase gene family and identification of alternatively spliced $X$ gene transcripts

Sargent, Carole; Affara, N

University of Cambridge, Department of Pathology, Tennis Court Road, Cambridge, CB2 1QP

Isolated glycerol kinase deficiency can be benign (adult form) or present with a variety of symptoms in the pre-school age group (juvenile form) which range from mild to severe. In order to find mutations resposible for the juvenile form we have analysed the gene family including the structure of the X-linked locus. In addition, we have demonstrated the presence of four isoforms of the $X$ transcript in a range of foetal tissues, with a different pattern of expression in adrenal and liver. One of the alternatively spliced exons may encode a sequence which is important for the correct compartmentation of the protein within the cell, and this is being investigated using the GFP reporter system. SSCP analysis and RT-PCR are being employed to study the gene and transcripts from patient samples, and so far one point mutation has been confirmed. This results in an amino acid substitution in one of the ATP binding domains, introducing a charged residue at a position which is non-polar in all glycerol kinases reported to date.

\subsection{2}

The Drosophila Developmental Gene Fat Facets Has a Human Homologue in Xp11.4 which Escapes Xinactivation and Has Related Sequences on Yq11.2. Michael H. Jones, Robert A. Furlong, Heather Burkin, I. Jennifer Chalmers, Graeme M. Brown, Omar Khwaja, Nabeel A. Affara

University of Canbridge Department of Pathology; Tennis Court Road; Cambridge; CB2 1QP.

Systematic sequence analysis of CDNA clones to generate ESTs has proved to be an effective means of identifying human transcripts that share homology with genes described in other species. This can give an indication of function and when combined with mapping to a particular chromosome region, it is possible to identify ESTs that are candidates for particular disease loci. The testis EST 221 detects similarity to the drosophila developmental gene fatfacets (faf). In dosophila, mutations of the faf gene are associated with two phenotypes. First, the gene is essential for normal oogenesis and, second, the gene product influences the fate of cells destined to become photoreceptors in the developing compound eye. This paper describes the analysis of the human $X$-linked homologue of the faf gene, a member of the deubiquitinating enzyme gene family. The gene is $X Y$ homologous, mapping to $X p 11.4$ and $Y q 11.2$ and these have been named DFFRX and DFFRY. Transcription occurs from the $X$ and $Y$ loci in both human adult and embryonic tissues and the gene on the $X$ has been shown to escape Xinactivation. The location of DFFRX in proximal Xp (Xp11.4), its escape from Xinactivation and the importance of faf for oocyte proliferation in drosophila raises the intriguing possibility that DFFRX is a candidate for the gonadal degeneration observed in Turner syncrome provoked by a failure of oocytes to proliferate and develop.

\subsection{4}

Analysis of the 1A1.3B gene and the adjacent breast cancer susceptibility gene, BRCA1, in mouse

Chambers, Julie; Griffiths, B; Nicolai, H; Brown, M; Xu, C-F; Solomon, E Somatic Cell Genetics, Imperial Cancer Research Fund, 44 Lincoln's Inn Fields, London, WC2A 3PX

The human 1A1.3B gene was isolated by expression cloning using antisera to CA125, an ovarian tumour antigen used in the management of ovarian cancer.1A1.3B is a novel gene but it is not clear whether $1 \mathrm{A1} .3 \mathrm{~B}$ is CA125. Mapping of this gene revealed that it lies adjacent to the breast cancer susceptibility gene, BRCA1. In order to investigate the function and importance of the 1A1.3B gene and its location adjacent to the BRCA1 gene, we have studied the conservation of this gene in a variety of species, by zoo blots, and in particular the mouse. We have isolated murine 1a1.3b cDNA and genomic clones. Translation of the CDNA sequence indicates that the protein is highly conserved being $89 \%$ similar and $84 \%$ identical to the human, suggesting that this gene has an important function. Analysis of murine genomic clones from this region indicates that the 1a1.3b gene lies head to head with the Brca1 gene about $1 \mathrm{~kb}$ apart, with the possibility that these genes may share a promoter or regulatory elements. Unlike in human, this region is not duplicated in mouse, indicating that the duplication is a recent event.

\subsection{5}

Truncated endothelin receptor $A$ transcript in human melanoma cell lines

Zhang, Yufeng; Jeffery, S; Wilson, CA; Kaski, JC; Bennett, D; Easty, DJ; Thody, AJ"; Burchill, SA \#; Carter, ND

St George's Hospital Medical School, "University of Newcastle Upon Tyne; \# ICRF Unit, St James Hospital Leods

Endothelins (ET-1, ET-2 and ET-3) are small 21 residue peptides derived from proteolysis of a precursor protein. These peptides mediate their effects on differentiation and proliferation through two receptors, ETA and ETB, which have sequence similarity to the G-protein heptahelical receptor family. Growth of human melanocytes in culture is markedly stimulated by endothelins and the presence of ETB has been demonstrated on melanocytes. However, there are no reports of ETA gene expression on melanocytes or melanoma cells. In the present study, human melanoma cell lines (DX3K, HMB2, LT5.1, MEWO, VUP and T8) were analysed by RT-PCR for the expression of ETA mRNA. Using several pairs of primers which covered the total coding region, high levels of a PCR product lacking exon 3 and 4 were detected and confirmed by DNA sequencing. This mRNA was the predominant ETA receptor transcript detectable in melanoma cell lines, whereas similar RT-PCR preparations from vascular tissue, placenta, liver and heart consistently gave full length ETA receptor transcripts, containing exon 3 and 4, but with a minor fraction (about $5 \%$ ) representing truncated ETA. The role of this truncated receptor in the development of malignancy and its cellular function remain to be evaluated. 


\subsection{6}

Structure and function of the glucokinase regulatory

protein (GCKR) and ketohexokinase (KHK) genes

Bonthron, David1; Hayward, BE1; Dunlop, N1; Intody, S1; Leek, J2; Markham, AF'; Warner, JP1

'Human Genetics Unit, University of Edinburgh, Western General Hospital, Edinburgh EH4 2XU; 2 Molecular Medicine Unit, University of Leeds, St. James's University Hospital, Leods LS9 7TF

Null glucokinase mutations cause maturity onset diabetes of the young, reflecting the role of islet cell glucokinase as a component of the blood glucose "sensor" controlling insulin output. However, glucokinase is also subject to complex post-translational regulation, suggesting that other genetic lesions could affect glucokinase activity and predispose to type 2 diabetes. We have cloned the human glucokinase regulatory protein gene (GCKR). GCKR's inhibition of glucokinase is allosterically relieved by fructose-1-phosphate (F-1-P) binding to GCKR, and we have also cloned the gene encoding fructokinase (ketohexokinase, KHK), which generates F-1-P. A YAC contig has been assembled, physically linking KHK and GCKR in chromosome 2p23. KHK has nine exons, two of which (3a and 3c) are alternatively spliced, generating two enzymatically active KHK isoforms. This alternative splicing is evolutionarily conserved and tissue specific. GCKR is encoded by a 19 exon gene, transcribed away from KHK. Intron 16 is of unknown size, but the rest of the gene spans $16 \mathrm{~kb}$. Exonic polymorphisms within KHK and GCKR, and a CA repeat upstream of KHK, have been identified; by linkage, the region maps between D2S220 and D2S165. Association, linkage, and mutation screening of GCKR exons in type 2 diabetes are now possible.

\subsection{7}

\section{Gender differences in age-of-onset of Huntington's} disease related to CAG repeat length and apolipoprotein E genotype.

Kehoe, Patrick (182); Krawczak, M1; Snell RG'; Liddell, M(182); Harper, PS1; Owen, M(182); Jones AL 1

I Institute of Medical Genetics; ${ }^{2}$ Dept Psychological Medicine, University of Wales College of Medicine, Heath Park, Cardiff CF4 4XN, U.K

Huntington's disease (HD) is an autosomal dominant neurodegenerative disease associated with the expansion of a (CAG)n repeat. The strongest phenotypic correlation in HD is the length of the expanded repeat varying inversely with age-of-onset $(\mathrm{AO})$ of the disease. We have previously shown in the Welsh population approximately $50 \%$ of the variation in $A O$ can be accounted for by the size of the expanded CAG allele and also that the shorter (normal) CAG repeat length affects AO. More detailed genetic analysis, using SAS, of $121 \mathrm{HD}$ patients from Wales, essentially reproduced the findings of the original study. Additionally, the length effect of the normal allele on $A O$ was shown to be limited to males $(r=$. $0.33, p=0.0138$ ). However, the most prominent sex difference with respect to our $A O$ correlate was the ratio of long to short repeat length ( $p$ $=0.0154$ ). Analysis of apolipoprotein $E$ (apoE) genotype showed three apoE genotypes occurring at frequencies allowing meaningful statistical analysis. Among the $\varepsilon 2 \varepsilon 3$ patients average $A O$ was 31.2 years for males and 48.1 years for females $(p<0.01)$. Genotypes $\varepsilon 3 \varepsilon 3$ and $\varepsilon 3 \varepsilon 4$ showed no gender differences in $A O$.

\subsection{8}

Membrane-associated huntingtin occurs in a higher molecular weight complex than soluble huntingtin Wood Jonathan D; Harper PS; Jones AL

Institute of Medical Genetics, University of Wales College of Medicine, Heath Park, Cardiff CF4 4XN, U.K

Huntington's disease $(\mathrm{HD})$ is an autosomal dominant neurodegenerative disease associated with the expansion of a (CAG)n repeat. The repeat gives rise to a polyglutamine stretch close to the $\mathrm{N}$-terminus of a $350 \mathrm{kDa}$ protein, huntingtin, which has no known function and few homologies in protein databases. We have previously found that huntingtin extracted from mammalian brain exists in both soluble and membrane-bound forms and that their relative distribution is species dependent. On native-PAGE the soluble form of huntingtin migrates with an Mr of $300-400 \mathrm{kDa}$. The membrane-associated form can be solubilised in 1.0\% Triton X-100 and migrates on native-PAGE with an $\mathrm{Mr}$ of $700-800 \mathrm{kDa}$ indicating that huntingtin exists as part of a larger complex when membrane-bound. In microtubule preparations huntingtin appears in the fraction containing polymerised microtubules from mouse, rat and human brain. In rat and human brain microtubule preparations huntingtin immunoreactivity also occurs in the membrane-containing fractions, in line with previous fractionation results. This evidence supports a possible role for huntingtin in cytoskeletal trafficking processes.

\subsection{9}

Two splice mutations in the ATR-X gene

Gibbons, Richard; Picketts, DJ; Bachoo, S; Higgs DR;

MRC Molecular Haematology Unit, Institute of Molecular Medicine, John Radcliffe Hospital, Oxford. OX3 9DU, UK

$X$-linked a thalassaemia mental retardation (ATR-X) syndrome is a severe form of syncromal mental retardation associated with a characteristic facial dysmorphism, genital abnormalities and a thalassaemia. The undertying gene has previously been identified and is a member of the SW1/SNF family of DNA dependent ATPases and putative helicases and includes proteins involved in transcriptional regulation. DNA repair and mitotic chromosome segregation. A systematic analysis of the mutations in 60 affected individuals is underway. To date a deletion, 2 nonsense mutations and 7 missense mutations have been identified. To facilitate the identification of the disease causing mutations in other patients we are currently cloning the full length CDNA sequence and characterising the genomic structure of this gene. Work to date has allowed us to identify two new splice mutations in the ATR-X gene. Genotype/phenotype correlations will be presented. 